\title{
Сингулярные расширения силлогистики Лукасевича
}

\author{
В. И. МАРКИН
}

\begin{abstract}
We implement two systems of syllogistic with singular terms that are the extensions of Łukasiewicz syllogistic. In the language of the first system singular terms can place only the subject position and singular propositions are of special type. In the language of the second one singular terms can place the subject as well as the predicate position and there are no special syllogistic constants for singular propositions. We prove the embedding of these singular syllogistics into the classical predicate calculus.
\end{abstract}

Ключевые слова: силлогистика, сингулярные термины, исчисление, семантика, формализация, погружающая операция

Широко известное силлогистическое исчисление, сформулированное Я. Лукасевичем в [1], является системой чистой позитивной силлогистики, поскольку в его языке содержатся нелогические символы только одного типа - общие термины (знаки множеств предметов), а среди логических символов отсутствуют терминообразующие операторы (например, терминное отрицание). Аксиомами силлогистики Лукасевича являются тавтологии классической логики высказываний (ЛВ) и формулы следующих типов:
A1. $(M \mathbf{a} P \& S \mathbf{a} M) \supset S \mathbf{a} P$
A4. $S \mathbf{i} S$,
A2. $(M \mathbf{a} P \& M \mathbf{i} S) \supset S \mathbf{i} P$,
A5. $S \mathbf{e} P \equiv \neg S \mathbf{i} P$,
A3. $S \mathbf{a} S$,
A6. $S \mathbf{o} P \equiv \neg S \mathbf{a} P$.

Сам Лукасевич последние две схемы аксиом трактовал как определения силлогистических констант е и о через, соответственно, константы $\mathbf{i}$ и $\mathbf{a}$. Единственное правило вывода в системе modus ponens. Понятия доказательства и теоремы обычные. Силлогистика Лукасевича в классификации В.А. Смирнова [3] получила название системы $\mathbf{C 4}$. 
Задача данной статьи заключается в том, чтобы предложить расширения силлогистики $\mathbf{C 4}$, в языке которых наряду с общими терминами содержались бы и сингулярные (единичные) термины - знаки отдельных предметов.

Существует, по крайней мере, два способа введения сингулярных терминов в язык силлогистики. Первый из них был использован Аристотелем, который в качестве примеров рассматривал силлогизмы с единичными высказываниями. Анализ этих примеров позволяет установить следующее. Сингулярные термины употребляются Аристотелем только в качестве субъектов высказываний, но никогда в качестве предикатов. Он не использует сингулярные термины в общих и частных высказываниях - высказываниях типа а, e, i и о. Иначе говоря, высказывания с сингулярными терминами представляют собой особые типы категорических высказываний «v есть $P »$ и «v не есть $P »$, не сводимые к другим типам - общим и частным высказываниям.

Другая трактовка роли сингулярных терминов в силлогистическом языке была характерна для У. Оккама. В противоположность Аристотелю Оккам использует сингулярные термины на местах как субъектов, так и предикатов высказываний. Причем единичные высказывания (высказывания с сингулярным субъектом) не рассматриваются им как высказывания особого типа, а представляют собой разновидность множественных (общих или частных) высказываний. При оккамовском подходе осмысленными оказываются высказывания вида «Всякий (некоторый) $v$ есть (не есть) $P$ ». Таким образом, второй способ введения сингулярных терминов в язык позитивной силлогистики не требует использования новых, отличных от $\mathbf{a}, \mathbf{e}, \mathbf{i}, \mathbf{o}$, силлогистических констант, допуская употребление на аргументных местах как универсалий, так и сингулярных терминов.

Современное формальное представление сингулярных расширений чистых позитивных силлогистик как в «аристотелевском», так и в «оккамовском» языке осуществлено в монографии [2] применительно к системе фундаментальной силлогистики $\mathbf{C \Phi}$ и к системе C2 В.А. Смирнова, которая считается наиболее адекватной формализацией силлогистики Аристотеля. В данной статье аналогичная задача будет решаться применительно 
к системе $\mathbf{C 4}$, формализующей традиционный вариант чистой позитивной силлогистики.

Сначала будем строить сингулярное расширение $\mathbf{C 4}$ в языке (условно будем называть аристотелевским), в котором предполагается, что сингулярные термины могут выступать только в качестве субъектов, а единичные высказывания образуют самостоятельные типы категорических высказываний. Нелогическими символами этого языка наряду с общими терминами (будем обозначать их в метаязыке буквами $S, P, Q, M, \ldots$ ) являются сингулярные термины (в качестве метапеременных по последним будем использовать знаки $\left.v, w, v_{1}, w_{1}, v_{2}, \ldots\right)$. Силлогистическими константами наряду со стандартными $(\mathbf{a}, \mathbf{e}, \mathbf{i}, \mathbf{o})$ будут новые константы ӓ (для единичноутвердительных высказываний) и ё (для единичноотрицательных высказываний). В алфавите содержатся также пропозициональные связки и скобки.

Определение формулы задается так:

1. Если $S$ и $P$ - общие термины, то выражения типов $S \mathbf{a} P$, $S \mathbf{i} P, S \mathbf{e} P, S \mathbf{o} P$ являются формулами;

2. Если $S$ - общий, а $v$ - сингулярный термин, то выражения типов $v \ddot{\mathbf{a}} S$ (читается: «v есть $S »)$ и $v \ddot{\mathbf{e}} S$ (читается: «v не есть $S \gg)$ суть формулы;

3. Если $\mathbf{A}$ и $\mathbf{B}-$ формулы, то $\neg \mathbf{A},(\mathbf{A} \& \mathbf{B}),(\mathbf{A} \vee \mathbf{B}),(\mathbf{A} \supset \mathbf{B})$, $(\mathbf{A} \equiv \mathbf{B})-$ формулы;

4. Ничто иное не есть формула.

В данном языке сформулируем исчисление $\mathbf{C} 4_{\mathbf{A}}^{\mathbf{C}}$, схемами аксиом которого являются:

$\mathbf{A}_{\mathbf{A}}^{\mathbf{C}} \mathbf{0 .}$ Схемы аксиом классического исчисления высказываний,

$\mathbf{A}_{\mathbf{A}}^{\mathbf{C}} \mathbf{1} . \quad(S \mathbf{a} P \& v \ddot{\mathbf{a}} S) \supset v \ddot{\mathbf{a}} P, \quad \mathbf{A}_{\mathbf{A}}^{\mathbf{C}} \mathbf{4} . \quad S \mathbf{o} S \equiv \neg S \mathbf{a} P$,

$\mathbf{A}_{\mathbf{A}}^{\mathbf{C}} \mathbf{2} . \quad(v \ddot{\mathbf{a}} P \& v \ddot{\mathbf{a}} S) \supset S \mathbf{i} P, \quad \mathbf{A}_{\mathbf{A}}^{\mathbf{C}} \mathbf{5} . \quad v \ddot{\mathbf{e}} S \equiv \neg v \ddot{\mathbf{a}} S$,

$\mathbf{A}_{\mathbf{A}}^{\mathbf{C}} \mathbf{3} . \quad S \mathbf{e} P \equiv \neg S \mathbf{i} P, \quad \mathbf{A}_{\mathbf{A}}^{\mathbf{C}} \mathbf{6} . \quad S \mathbf{i} S$,

а правилами вывода: 
R1. modus ponens,

R2. $\frac{\vdash(v \ddot{\mathbf{a}} S \& v \ddot{\mathbf{a}} P) \supset A}{\vdash S \mathbf{i} P \supset A}, \quad \mathbf{R 3} . \quad \frac{\vdash(v \ddot{\mathbf{a}} S \& v \ddot{\mathbf{e}} P) \supset A}{\vdash S \mathbf{o} P \supset A}$,

где сингулярный термин $v$ не содержится в формуле $\mathbf{A}$.

Покажем, что исчисление $\mathbf{C} \mathbf{4}_{\mathbf{A}}^{\mathbf{C}}$ представляет собой расширение силлогистики $\mathbf{C 4}$ :

METATЕОРЕМА 1. Всякая теорема системы $\mathbf{C 4}$ доказуема в исчислении $\mathbf{C} 4_{\mathbf{A}}^{\mathbf{C}}$.

\section{Доказательство.}

Достаточно продемонстрировать доказуемость в $\mathbf{C} 4_{\mathbf{A}}^{\mathbf{C}}$ всех аксиом системы C4.

Пропозициональные тавтологии доказуемы в $\mathbf{C 4}_{\mathbf{A}}^{\mathbf{C}}$ в силу $\mathbf{A}_{\mathbf{A}}^{\mathbf{C}} \mathbf{0}$.

A1. $(M \mathbf{a} P \& S \mathbf{a} M) \supset S \mathbf{a} P$

1. $(M \mathbf{a} P \& v \ddot{\mathbf{a}} S) \supset v \ddot{\mathbf{a}} P \quad \mathbf{A}_{\mathbf{A}}^{\mathbf{C}} \mathbf{1}$

2. $(S \ddot{\mathbf{a}} M \& v \ddot{\mathbf{a}} S) \supset v \ddot{\mathbf{a}} M \quad \quad \mathbf{A}_{\mathbf{A}}^{\mathbf{C}} \mathbf{1}$

3. $\quad(M \mathbf{a} P \& S \mathbf{a} M \& v \ddot{\mathbf{a}} S) \supset v \ddot{\mathbf{a}} P \quad 1,2 ;$ ЛB

4. $v \ddot{\mathbf{e}} P \equiv \neg v \ddot{\mathbf{a}} P \quad \mathbf{A}_{\mathbf{A}}^{\mathbf{C}} \mathbf{5}$

5. $\quad(v \ddot{\mathbf{a}} S \& v \ddot{\mathbf{e}} P) \supset \neg(M \mathbf{a} P \& S \mathbf{a} M) \quad 3,4 ; \pi \mathbf{B}$

6. $S \mathbf{o} P \supset \neg(M \mathbf{a} P \& S \mathbf{a} M) \quad$ 5; R3

7. $S \mathbf{o} P \equiv \neg S \mathbf{a} P \quad \mathbf{A}_{\mathbf{A}}^{\mathbf{C}} \mathbf{4}$

8. $(M \mathbf{a} P \& S \mathbf{a} M) \supset S \mathbf{a} P \quad 6,7 ;$ ЛB

A2. $(M \mathbf{a} P \& M \mathbf{i} S) \supset S \mathbf{i} P$

1. $(M \mathbf{a} P \& v \ddot{\mathbf{a}} S) \supset v \ddot{\mathbf{a}} P \quad \mathbf{A}_{\mathbf{A}}^{\mathbf{C}} \mathbf{1}$

2. $(v \ddot{\mathbf{a}} P \& v \ddot{\mathbf{a}} S) \supset S \mathbf{i} P \quad \quad \mathbf{A}_{\mathbf{A}}^{\mathbf{C}} \mathbf{2}$

3. $(\neg v \ddot{\mathbf{a}} P \& v \ddot{\mathbf{a}} M) \supset \neg M \mathbf{a} P \quad 1 ; \pi \mathbf{B}$

4. $\quad(v \ddot{\mathbf{a}} M \& v \ddot{\mathbf{a}} S) \supset(S \mathbf{i} P \vee \neg M \mathbf{a} P) \quad 2,3 ;$ ЛВ

5. $M \mathbf{i} S \supset(S \mathbf{i} P \vee \neg M \mathbf{a} P) \quad 4 ; \mathbf{R 2}$

6. $(M \mathbf{a} P \& M \mathbf{i} S) \supset S \mathbf{i} P \quad 5 ;$ ЛВ

A3. $S \mathbf{a} S$

1. $v \ddot{\mathbf{e}} S \equiv \neg v \ddot{\mathbf{a}} S \quad \mathbf{A}_{\mathbf{A}}^{\mathbf{C}} \mathbf{5}$

2. $\quad(v \ddot{\mathbf{a}} S \& v \ddot{\mathbf{e}} S) \supset S \mathbf{a} S \quad 1 ;$ ЛB 


\section{3. $S \mathbf{o} S \supset S \mathbf{a} S \quad 2 ; \mathbf{R 3}$ \\ 4. $S \mathbf{o} S \equiv \neg S \mathbf{a} S \quad \mathbf{A}_{\mathbf{A}}^{\mathbf{C}} \mathbf{4}$ \\ 5. $S \mathbf{a} S \quad 3,4 ;$ ЛВ}

Аксиомы видов $\mathbf{A} 4, \mathbf{A 5}$ и $\mathbf{A 6}$ системы $\mathbf{C 4}$ доказуемы в $\mathbf{C} 4 \mathbf{A}$, поскольку они являются соответственно аксиомами $\mathbf{A}_{\mathbf{A}}^{\mathbf{C}} \mathbf{6}, \mathbf{A}_{\mathbf{A}}^{\mathbf{C}} \mathbf{3}$ и $\mathbf{A}_{\mathbf{A}}^{\mathbf{C}} \mathbf{4}$ последней системы.

Q.E.D.

Далее мы покажем, что силлогистика $\mathbf{C} \mathbf{4}_{\mathbf{A}}^{\mathbf{C}}$ погружается в классическое одноместное исчисление предикатов. С этой целью первоначально демонстрируется погружаемость $\mathbf{C} \mathbf{4}_{\mathbf{A}}^{\mathbf{C}}$ в другую систему сингулярной позитивной силлогистики, а именно в систему $\mathbf{C} \boldsymbol{\Phi}_{\mathbf{A}}^{\mathbf{C}}-$ «фундаментальный» вариант силлогистики указанного типа.

Исчисление $\mathbf{C} \boldsymbol{\Phi}_{\mathbf{A}}^{\mathbf{C}}$ получается за счет отбрасывания из аксиомной схемы $\mathbf{A}_{\mathbf{A}}^{\mathbf{C}} \mathbf{6}-S \mathbf{i} S$. Это исчисление подробно исследовано нами в [2]. В частности, для него была сформулирована адекватная семантика.

Моделъю является пара $\langle\mathbf{D}, \varphi\rangle$, где $\mathbf{D} \neq \varnothing, \varphi$ приписывает значения сингулярным и общим терминам следующим образом: $\varphi(v) \in \mathbf{D}, \varphi(S) \subseteq \mathbf{D}$. Условия истинности элементарных формул в модели $<\mathbf{D}, \varphi>$ таковы:

И1. $|S \mathbf{a} P|_{\varphi}=1$, е.т.е. $\varphi(S) \subseteq \varphi(P)$,

И2. $|S \mathbf{i} P|_{\varphi}=1$, е.т.е. $\varphi(S) \cap \varphi(P) \neq \varnothing$,

И3. $|S \mathbf{e} P|_{\varphi}=1$, е.т.е. $\varphi(S) \cap \varphi(P)=\varnothing$,

И4. $|S \mathbf{o} P|_{\varphi}=1$, е.т.е. $\varphi(S) \backslash \varphi(P) \neq \varnothing$,

И5. $|v \ddot{a} P|_{\varphi}=1$, е.т.е. $\varphi(S) \in \varphi(P)$,

И6. $|v \ddot{\mathbf{e}} P|_{\varphi}=1$, е.т.е. $\varphi(S) \notin \varphi(P)$.

Условия истинности для сложных формул, понятие истинности в модели и $\mathbf{C} \boldsymbol{\Phi}_{\mathbf{A}}^{\mathbf{C}}$-общезначимости стандартные.

В [2] доказаны непротиворечивость и полнота системы $\mathbf{C} \boldsymbol{\Phi}_{\mathbf{A}}^{\mathbf{C}}$ относительно приведенной семантики. Отсюда следует справедливость утверждения:

МЕТАТЕОРЕМА 2. Всякая формула доказуема в исчислении $\mathbf{C} \boldsymbol{\Phi}_{\mathbf{A}}^{\mathbf{C}}$, если и только если она является $\mathbf{C} \boldsymbol{\Phi}_{\mathbf{A}}^{\mathbf{C}}$-общезначимой. 
Был также задан «фундаментальный» перевод * формул языка сингулярной позитивной силлогистики аристотелевского типа на язык классического исчисления предикатов:

$$
\begin{array}{lll}
(S \mathbf{a} P)^{*}=\forall x(S x \supset P x), & & (S \mathbf{e} P)^{*}=\forall x(S x \supset \neg P x), \\
(S \mathbf{i} P)^{*}=\exists x(S x \& P x), & & (S \mathbf{o} P)^{*}=\exists x(S x \& \neg P x), \\
(v \ddot{\mathbf{a}} S)^{*}=S v, & & (v \ddot{\mathbf{e} S})^{*}=\neg S v, \\
(\neg \mathbf{A})^{*}=\neg \mathbf{A}^{*}, & & (\mathbf{A} \nabla \mathbf{B})^{*}=\mathbf{A}^{*} \nabla \mathbf{B}^{*},
\end{array}
$$

где $\nabla$ есть любая бинарная пропозициональная связка.

Доказана метатеорема о погружаемости системы $\mathbf{C} \boldsymbol{\Phi}_{\mathbf{A}}^{\mathbf{C}}$ в классическое одноместное исчисление предикатов (ИП):

METATЕОРЕМА 3. Всякая формула А доказуема в исчислении $\mathbf{C \Phi}_{\mathbf{A}}^{\mathbf{C}}$, если и только если ее перевод $\mathbf{A}^{*}$ доказуем в ИП.

Для силлогистики $\mathbf{C} \mathbf{4}_{\mathbf{A}}^{\mathbf{C}}$ зададим другую функцию перевода $\Theta$ на язык ИП, определяемую с использованием функции *:

$$
\Theta(\mathbf{A})=\left(\exists x S_{1} x \& \ldots \& \exists x S_{n} x\right) \supset \mathbf{A}^{*},
$$

где $S_{1}, \ldots, S_{n}-$ список всех различных общих терминов в составе формулы $\mathbf{A}$.

Перевод $\Theta$ указывает на то, что любое силлогистическое утверждение в $\mathbf{C} \mathbf{4}_{\mathbf{A}}^{\mathbf{C}}$ содержит характерную для традиционной силлогистики пресуппозицию о непустоте всех входящих в него общих терминов. Что же касается сингулярных терминов, то нет никакой необходимости эксплицитно выражать для них подобную пресуппозицию, поскольку утверждение о непустоте всех сингулярных терминов - один из исходных постулатов классического исчисления предикатов.

Перед обоснованием тезиса о погружаемости $\mathbf{C} 4_{\mathbf{A}}^{\mathbf{C}}$ в ИП посредством $\Theta$ докажем, что эта система погружается в фундаментальную сингулярную позитивную силлогистику $\mathbf{C} \boldsymbol{\Phi}_{\mathbf{A}}^{\mathbf{C}}$ посредством следующей операции:

$$
\omega_{1}(\mathbf{A})=\left(S_{1} \mathbf{i} S_{1} \& \ldots \& S_{n} \mathbf{i} S_{n}\right) \supset \mathbf{A},
$$

где $S_{1}, \ldots, S_{n}$ - список всех содержащихся в А общих терминов. 
METATEOPEMA 4. Перевод $\omega_{1}$ погружает $\mathbf{C} 4_{\mathbf{A}}^{\mathbf{C}}$ в $\mathbf{C} \boldsymbol{\Phi}_{\mathbf{A}}^{\mathbf{C}}$.

Доказательство. В доказательстве метатеоремы будем использовать следующий критерий погружаемости одного исчисления в другое, предложенный В.А. Смирновым [4]:

Исчисление $\mathbf{S}_{\mathbf{1}}$ погружсается в исчисление $\mathbf{S}_{\mathbf{2}}$ посредством функиии $\psi_{1}$ (из множества формул $\mathbf{S}_{\mathbf{1}}$ во множество формул $\mathbf{S}_{\mathbf{2}}$ ), если и только если (1) для каждой формулы языка $\mathbf{S}_{\mathbf{1}}$ имеет место: если $\mathbf{S}_{\mathbf{1}} \vdash \mathbf{A}$, то $\mathbf{S}_{\mathbf{2}} \vdash \psi_{\mathbf{1}}(\mathbf{A})$, и существует функиия $\psi_{2}$ из множества формул $\mathbf{S}_{\mathbf{2}}$ во множество формул $\mathbf{S}_{\mathbf{1}}$, такая что (2) для каждой формуль $\mathbf{A}$ языка $\mathbf{S}_{\mathbf{2}}$ имеет место: если $\mathbf{S}_{\mathbf{2}} \vdash \mathbf{A}$, то $\vdash \psi_{2}(\mathbf{A})$, $u(\mathbf{3}) \mathbf{S}_{\mathbf{1}} \vdash \mathbf{A} \equiv \psi_{2}\left(\psi_{1}(\mathbf{A})\right)$ для каждой формуль $\mathbf{A}$ языка $\mathrm{S}_{1}$.

Продемонстрируем сначала, что $\omega_{1}$-переводы всех теорем $\mathbf{C} \mathbf{4}_{\mathbf{A}}^{\mathbf{C}}$ доказуемы в фундаментальной силлогистике. Рассмотрим доказательство $\mathbf{C}_{1}, \ldots, \mathbf{C}_{k}$ произвольной теоремы системы $\mathbf{C} \mathbf{4}_{\mathbf{A}}^{\mathbf{C}}$ и методом возвратной индукции покажем для каждого $\mathbf{C}_{i}$, что $\mathbf{C} \boldsymbol{\Phi}_{\mathbf{A}}^{\mathbf{C}} \vdash \omega_{1}\left(\mathbf{C}_{i}\right)$.

Пусть $\mathbf{C}_{i}-$ аксиома $\mathbf{C} 4_{\mathbf{A}}^{\mathbf{C}}$. Аксиомы $\mathbf{A}_{\mathbf{A}}^{\mathbf{C}} \mathbf{1}-\mathbf{A}_{\mathbf{A}}^{\mathbf{C}} \mathbf{5}$ системы $\mathbf{C} 4_{\mathbf{A}}^{\mathbf{C}}$ являются также аксиомами $\mathbf{C} \boldsymbol{\Phi}_{\mathbf{A}}^{\mathbf{C}}$. Поэтому их $\omega_{1}$-переводы выводятся в последней системе из соответствующих аксиом с использованием закона утверждения консеквента. $\omega_{1}$-перевод аксиомы $\mathbf{A}_{\mathbf{A}}^{\mathbf{C}} \mathbf{6}$ представляет собой формулу $S \mathbf{i} S \supset S \mathbf{i} S$, которая является классической тавтологией.

Пусть $\mathbf{C}_{i}$ получено из $\mathbf{C}_{j} \supset \mathbf{C}_{i}$ и $\mathbf{C}_{j}$ по modus ponens. Пусть $M_{1}, \ldots, M_{r}-$ список общих терминов, входящих в $\mathbf{C}_{j}$, но не входящих в $\mathbf{C}_{i} ; P_{1}, \ldots, P_{m}-$ список общих терминов, входящих и в $\mathbf{C}_{j}$, и в $\mathbf{C}_{i}$, а $Q_{1}, \ldots, Q_{n}-$ список общих терминов, не входящих в $\mathbf{C}_{j}$, но входящих в $\mathbf{C}_{i}$. Ясно, что по отдельности каждый из трех списков может, в принципе, оказаться пустым, но по крайней мере один - первый или второй, а также второй или третий - обязательно непуст. Обозначим через $\mathbf{K}_{M}$ формулу $M_{1} \mathbf{i} M_{1} \& \ldots \& M_{r} \mathbf{i} M_{r}$, через $\mathbf{K}_{P}-$ формулу $P_{1} \mathbf{i} P_{1} \& \ldots \& P_{m} \mathbf{i} P_{m}$, и через $\mathbf{K}_{Q}-$ формулу $Q_{1} \mathbf{i} Q_{1} \& \ldots \& Q_{n} \mathbf{i} Q_{n}$.

Очевидно, что $\omega_{1}\left(\mathbf{C}_{j}\right)=\left(\mathbf{K}_{M} \& \mathbf{K}_{P}\right) \supset \mathbf{C}_{j}, \omega_{1}\left(\mathbf{C}_{i}\right)=\left(\mathbf{K}_{P} \&\right.$ $\left.\mathbf{K}_{Q}\right) \supset \mathbf{C}_{i}$, a $\omega_{1}\left(\mathbf{C}_{j} \supset \mathbf{C}_{i}\right)=\left(\mathbf{K}_{M} \& \mathbf{K}_{P} \& \mathbf{K}_{Q}\right) \supset\left(\mathbf{C}_{j} \supset \mathbf{C}_{i}\right)$. 
Согласно индуктивному допущению, $\omega_{1}\left(\mathbf{C}_{j} \supset \mathbf{C}_{i}\right)$ и $\omega_{1}\left(\mathbf{C}_{j}\right)$ доказуемы в $\mathbf{C} \boldsymbol{\Phi}_{\mathbf{A}}^{\mathbf{C}}$. Покажем, что в таком случае теоремой этой системы будет и формула $\omega_{1}\left(\mathbf{C}_{i}\right)$.

Из доказуемости $\left(\mathbf{K}_{M} \& \mathbf{K}_{P}\right) \supset \mathbf{C}_{j}$ и $\left(\mathbf{K}_{M} \& \mathbf{K}_{P} \& \mathbf{K}_{Q}\right) \supset\left(\mathbf{C}_{j} \supset\right.$ $\left.\mathbf{C}_{i}\right)$ в силу законов логики высказываний вытекает доказуемость в $\mathbf{C} 4_{\mathbf{A}}^{\mathbf{C}}$ формулы $\mathbf{K}_{M} \supset\left(\left(\mathbf{K}_{P} \& \mathbf{K}_{Q}\right) \supset \mathbf{C}_{i}\right)$, т.е. $\mathbf{K}_{M} \supset \omega_{1}\left(\mathbf{C}_{i}\right)$.

Если список $M_{1}, \ldots, M_{r}$ пуст, то последнее выражение есть просто $\omega_{1}\left(\mathbf{C}_{i}\right)$, и мы уже получили утверждение о доказуемости $\omega_{1}$-перевода $\mathbf{C}_{i}$.

В случае непустоты указанного списка продолжим рассуждение методом «от противного». Допустим, что $\omega_{1}\left(\mathbf{C}_{i}\right)$ недоказуема в $\mathbf{C} \boldsymbol{\Phi}_{\mathbf{A}}^{\mathbf{C}}$. В силу Метатеоремы 2 данная формула не является $\mathbf{C} \Phi_{\mathbf{A}}^{\mathbf{C}}$-общезначимой, т.е. существует модель $\langle\mathbf{D}, \varphi>$, в которой она принимает значение $\mathbf{0}$. Рассмотрим модель $<\mathbf{D}, \varphi^{\prime}>$, где ' всем терминам, отличным от $M_{1}, \ldots, M_{r}$, приписывает те же самые значения, что и $\varphi$, а общим терминам из списка $M_{1}, \ldots, M_{r}$ сопоставляет в качестве значений множество $\mathbf{D}: \varphi^{\prime}\left(M_{1}\right)=$ $\varphi^{\prime}\left(M_{2}\right)=\cdots=\varphi^{\prime}\left(M_{r}\right)=\mathbf{D}$. В модели $<\mathbf{D}, \varphi^{\prime}>$ каждая из формул $M_{1} \mathbf{i} M_{1}, \ldots, M_{r} \mathbf{i} M_{r}$ примет значение $\mathbf{1}$ в силу того, что множество $\mathbf{D}$ непусто. Поэтому и конъюнкция этих формул $\mathbf{K}_{M}$ истинна в рассматриваемой модели. Что же касается формулы $\omega_{1}\left(\mathbf{C}_{i}\right)$, то поскольку она не содержит терминов $M_{1}, \ldots, M_{r}$, значение ее в $<\mathbf{D}, \varphi^{\prime}>$ останется тем же самым, что и в модели $<\mathbf{D}, \varphi>$, т.е. она в обеих моделях принимает значение $\mathbf{0}$. Из сказанного следует, что формула $\mathbf{K}_{M} \supset \omega_{1}\left(\mathbf{C}_{i}\right)$ ложна в $\left\langle\mathbf{D}, \varphi^{\prime}\right\rangle$, и потому она не является $\mathbf{C} \boldsymbol{\Phi}_{\mathbf{A}}^{\mathbf{C}}$-общезначимой. Отсюда в силу Метатеоремы 2 получаем, что $\mathbf{K}_{M} \supset \omega_{1}\left(\mathbf{C}_{i}\right)$ недоказуема в $\mathbf{C} \Phi_{\mathbf{A}}^{\mathbf{C}}$, что противоречит выведенному ранее из индуктивного предположения следствию. Поэтому допущение о недоказуемости $\omega_{1}\left(\mathbf{C}_{i}\right)$ неверно. Следовательно, эта формула является теоремой $\mathbf{C} \boldsymbol{\Phi}_{\mathbf{A}}^{\mathbf{C}}$.

Пусть $\mathbf{C}_{i}$ получено из $\mathbf{C}_{j}$ по правилу $\mathbf{R 2}$. Тогда $\mathbf{C}_{j}=(v \ddot{\mathbf{a}} S \&$ $v \ddot{\mathbf{a}} P) \supset \mathbf{A}$, а $\mathbf{C}_{i} \mp S \mathbf{i} P \supset \mathbf{A}$. Множества общих терминов в $\mathbf{C}_{j}$ и $\mathbf{C}_{i}$ совпадают.

1. $\mathbf{C} \Phi_{\mathbf{A}}^{\mathbf{C}} \vdash \omega_{1}((v \ddot{\mathbf{a}} S \& v \ddot{\mathbf{a}} P) \supset \mathbf{A})$ инд. доп.

2. $\mathbf{C} \Phi_{\mathbf{A}}^{\mathbf{C}} \vdash\left(S_{1} \mathbf{i} S_{1} \& \ldots \& S_{n} \mathbf{i} S_{n}\right) \supset((v \ddot{\mathbf{a}} S \& v \ddot{\mathbf{a}} P) \supset \mathbf{A}) \quad$ 1, опр. $\omega_{1}$

3. $\mathbf{C} \Phi_{\mathbf{A}}^{\mathbf{C}} \vdash(v \ddot{\mathbf{a}} S \& v \ddot{\mathbf{a}} P) \supset\left(\left(S_{1} \mathbf{i} S_{1} \& \ldots \& S_{n} \mathbf{i} S_{n}\right) \supset \mathbf{A}\right) \quad 2 ;$ ЛB 
4. $\mathbf{C} \Phi_{\mathbf{A}}^{\mathbf{C}} \vdash S \mathbf{i} P \supset\left(\left(S_{1} \mathbf{i} S_{1} \& \ldots \& S_{n} \mathbf{i} S_{n}\right) \supset \mathbf{A}\right)$

5. $\mathbf{C} \boldsymbol{\Phi}_{\mathbf{A}}^{\mathbf{C}} \vdash\left(S_{1} \mathbf{i} S_{1} \& \ldots \& S_{n} \mathbf{i} S_{n}\right) \supset(S \mathbf{i} P \supset \mathbf{A})$

6. $\mathbf{C} \boldsymbol{\Phi}_{\mathbf{A}}^{\mathbf{C}} \vdash \omega_{1}(S \mathbf{i} P \supset \mathbf{A})$

Случай, когда $\mathbf{C}_{i}$ получено по правилу $\mathbf{R} \mathbf{3}$, рассматривается аналогично.

Итак, $\omega_{1}$-перевод любой теоремы $\mathbf{C} 4_{\mathbf{A}}^{\mathbf{C}}$ доказуем в системе $\mathbf{C} \boldsymbol{\Phi}_{\mathbf{A}}^{\mathbf{C}}$. Рассмотрение первой части критерия В.А. Смирнова завершено.

Зададим теперь обратный перевод $\omega_{2}$ из $\mathbf{C} \boldsymbol{\Phi}_{\mathbf{A}}^{\mathbf{C}}$ в $\mathbf{C} 4_{\mathbf{A}}^{\mathbf{C}}$ :

$$
\omega_{2}(\mathbf{A})=\mathbf{A} \text {. }
$$

Поскольку исчисление $\mathbf{C} \boldsymbol{\Phi}_{\mathbf{A}}^{\mathbf{C}}$ является подсистемой $\mathbf{C} 4_{\mathbf{A}}^{\mathbf{C}}, \omega_{2}-$ перевод любой теоремы $\mathbf{C} \boldsymbol{\Phi}_{\mathbf{A}}^{\mathbf{C}}$ доказуем в $\mathbf{C} \mathbf{4}_{\mathbf{A}}^{\mathbf{C}}$.

Перейдем к рассмотрению третьей, последней части критерия В.А. Смирнова. Необходимо показать, что для произвольной формулы $\mathbf{A}$ языка систем $\mathbf{C} 4_{\mathbf{A}}^{\mathbf{C}}$ и $\mathbf{C} \boldsymbol{\Phi}_{\mathbf{A}}^{\mathbf{C}}$ верно утверждение:

$$
\mathbf{C 4}_{\mathbf{A}}^{\mathbf{C}} \vdash \mathbf{A} \equiv \omega_{2}\left(\omega_{1}(\mathbf{A})\right) .
$$

Поскольку $\omega_{2}\left(\omega_{1}(\mathbf{A})\right)=\omega_{1}(\mathbf{A})=\left(S_{1} \mathbf{i} S_{1} \& \ldots \& S_{n} \mathbf{i} S_{n}\right) \supset \mathbf{A}$, нам требуется доказать в $\mathbf{C} 4_{\mathbf{A}}^{\mathbf{C}}$ формулу $\mathbf{A} \equiv\left(\left(S_{1} \mathbf{i} S_{1} \& \ldots\right.\right.$ $\left.\left.\& S_{n} \mathbf{i} S_{n}\right) \supset \mathbf{A}\right):$

1. $\mathbf{A} \supset\left(\left(S_{1} \mathbf{i} S_{1} \& \ldots \& S_{n} \mathbf{i} S_{n}\right) \supset \mathbf{A}\right) \quad$ тавтология ЛВ

2. $S_{1} \mathbf{i} S_{1} \& \ldots \& S_{n} \mathbf{i} S_{n} \quad$ из $\mathbf{A}_{\mathbf{A}}^{\mathbf{C}} \mathbf{6} ;$ ЛВ

3. $\left(\left(S_{1} \mathbf{i} S_{1} \& \ldots \& S_{n} \mathbf{i} S_{n}\right) \supset \mathbf{A}\right) \supset \mathbf{A} \quad 2 ;$ ЛB

4. $\mathbf{A} \equiv\left(\left(S_{1} \mathbf{i} S_{1} \& \ldots \& S_{n} \mathbf{i} S_{n}\right) \supset \mathbf{A}\right) \quad 1,3 ;$ ЛB

Таким образом, все три части критерия погружаемости выполняются, следовательно, перевод $\omega_{1}$ погружает $\mathbf{C} 4_{\mathbf{A}}^{\mathbf{C}}$ в $\mathbf{C} \boldsymbol{\Phi}_{\mathbf{A}}^{\mathbf{C}}$. Q.E.D.

Перейдем к доказательству основной метатеоремы:

METATEOPEMA 5. Перевод $\Theta$ погружает $\mathbf{C 4} \underset{\mathbf{A}}{\mathbf{C}}$ в ИП. 


\section{Доказательство.}

Из доказанных ранее утверждений о погружаемости системы $\mathbf{C} \Phi_{\mathbf{A}}^{\mathbf{C}}$ в ИП посредством операции * (Метатеорема 3) и о погружаемости $\mathbf{C} 4_{\mathbf{A}}^{\mathbf{C}}$ в свою подсистему $\mathbf{C} \boldsymbol{\Phi}_{\mathbf{A}}^{\mathbf{C}}$ (Метатеорема 4) посредством функции $\omega_{1}$ следует, что силлогистика $\mathbf{C} 4_{\mathbf{A}}^{\mathbf{C}}$ погружается в ИП посредством композиции переводов $\omega_{1}{ }^{*}$. Остается показать, что эта композиция равносильна в ИП переводу $\Theta$ :

$$
\begin{gathered}
\omega_{1}(\mathbf{A})^{*}=\left(\left(S_{1} \mathbf{i} S_{1} \& \ldots \& S_{n} \mathbf{i} S_{n}\right) \supset \mathbf{A}\right)^{*}=\left(S_{1} \mathbf{i} S_{1} \& \ldots\right. \\
\left.\& S_{n} \mathbf{i} S_{n}\right)^{*} \supset \mathbf{A}^{*}=\left(\exists x ( S _ { 1 } x \& S _ { 1 } x ) \& \ldots \& \exists x \left(S_{n} x \&\right.\right. \\
\left.\left.\& S_{n} x\right)\right) \supset \mathbf{A}^{*} \equiv\left(\exists x S_{1} x \& \ldots \& \exists x S_{n} x\right) \supset \mathbf{A}^{*}=\Theta(\mathbf{A}) .
\end{gathered}
$$

Q.E.D.

Таким образом, построено расширение силлогистики Лукасевича за счет введения в язык сингулярных терминов и оперирования ими в аристотелевском стиле.

Перейдем теперь к формулировке другого сингулярного расширения чистой позитивной силлогистики $\mathbf{C 4}$, основанного на двух описанных выше оккамовских принципах употребления сингулярных терминов: 1) сингулярные термины допускаются как на места субъектов, так и на места предикатов; 2) высказывания с сингулярным субъектом не рассматриваются в качестве самостоятельного типа категорических высказываний, а могут быть либо общими, либо частными. Таким образом, здесь разрешается квантификация сингулярных терминов: «Всякий (некоторый) $v$ есть (не есть) $P$ ». В связи с этим необходимо пересмотреть их семантику, предположив, что значениями сингулярных терминов являются теперь не индивиды, а одноэлементные множества.

Язык сингулярной позитивной силлогистики, построенный на основе указанных выше принципов, будем условно называть оккамовским. В алфавит оккамовского языка, как и в алфавит языка аристотелевского типа, входят бесконечные списки общих и сингулярных терминов, пропозициональные связки и скобки. Но в данном языке содержатся лишь стандартные силлогистические константы $\mathbf{a}, \mathbf{e}, \mathbf{i}$ и о. 
Здесь существенным оказывается введение понятия силлогистического термина:

1. Всякий общий термин является силлогистическим термином;

2. Всякий сингулярный термин является силлогистическим термином;

3. Ничто иное не является силлогистическим термином.

Элементарными формулами в оккамовском языке являются выражения видов $S \mathbf{a} P, S \mathbf{e} P, S \mathbf{i} P$ и $S \mathbf{o} P$, где $S$ и $P$ - силлогистические термины любого из двух типов. Сложные формулы образуются из других формул с помощью пропозициональных связок.

В [2] было осуществлено расширение в данном языке двух систем чистой позитивной силлогистики - $\mathbf{C \Phi}$ и $\mathbf{C 2}$. Исчисление $\mathbf{C} \boldsymbol{\Phi}_{\mathbf{O}}^{\mathbf{C}}$, формализующее «фундаментальный» вариант сингулярной позитивной силлогистики, содержит следующие схемы аксиом:

$\mathbf{A}_{\mathbf{O}}^{\mathbf{C}} \mathbf{0}$. Схемы аксиом классического исчисления высказываний,
$\mathbf{A}_{\mathbf{O}}^{\mathbf{C}} \mathbf{1} . \quad v \mathbf{a} v$,
$\mathbf{A}_{\mathbf{O}}^{\mathbf{C}} 4 . \quad(v \mathbf{a} P \& v \mathbf{a} S) \supset S \mathbf{i} P$,
$\mathbf{A}_{\mathbf{O}}^{\mathbf{C}}$ 2. $v \mathbf{a} w \supset w \mathbf{a} v$,
$\mathbf{A}_{\mathbf{O}}^{\mathbf{C}} 5 . \quad S \mathbf{e} P \equiv \neg S \mathbf{i} P$,
$\mathbf{A}_{\mathbf{O}}^{\mathbf{C}}$ 3. $(M \mathbf{a} P \& v \mathbf{a} M) \supset v \mathbf{a} P$,
$\mathbf{A}_{\mathbf{O}}^{\mathbf{C}} 6 . \quad S \mathbf{o} P \equiv \neg S \mathbf{a} P$.

В системе $\mathbf{C} \Phi_{\mathrm{O}}^{\mathbf{C}}$ три правила вывода:

П1. modus ponens,

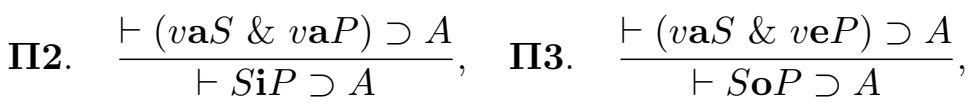

где $v$ не содержится в заключении. $S, P$ и $M$ в формулировках аксиомных схем и правил - любые силлогистические термины (как общие, так и сингулярные), $v$ и $w$ - сингулярные термины.

В [2] сформулирована следующая семантика силлогистики $\mathbf{C} \boldsymbol{\Phi}_{\mathbf{O}}^{\mathbf{C}}$. Моделью является пара $\langle\mathbf{D}, \varphi\rangle$, где $\mathbf{D} \neq \varnothing$, $\varphi(v) \in \mathbf{D}$, а $\varphi(S) \subseteq \mathbf{D}$, где $S-$ общий термин. Далее в модели $\langle\mathbf{D}, \varphi\rangle$ определяется новая функция $\sigma$, приписывающая значения любым силлогистическим терминам - как сингулярным, так и общим. Такими значениями должны быть подклассы D. Сингулярному термину $v$ функция приписывает класс, единственным элементом которого является объект, сопоставленный 
$v$ посредством $\varphi$, т.е. $\sigma(v)=\{\varphi(v)\}$. А общему термину $S$ функция $\sigma$ сопоставит тот же класс, что и $\varphi$, т.е. $\sigma(S)=\varphi(S)$. Условия истинности формул типа $S \mathbf{a} P, S \mathbf{i} P, S \mathbf{e} P, S \mathbf{o} P$ оккамовского языка в модели $\langle\mathbf{D}, \varphi>$ отличаются от определений И1-И4 заменой $\varphi$ на $\sigma$ в определяющих частях:

У1. $|S \mathbf{a} P|_{\varphi}=1$, е.т.е. $\sigma(S) \subseteq \sigma(P)$

У2. $|S \mathbf{i} P|_{\varphi}=1$, е.т.е. $\sigma(S) \cap \sigma(P) \neq \varnothing$

У3. $|S \mathbf{e} P|_{\varphi}=1$, е.т.е. $\sigma(S) \cap \sigma(P)=\varnothing$

У4. $|S \mathbf{o} P|_{\varphi}=1$, е.т.е. $\sigma(S) \backslash \sigma(P) \neq \varnothing$

Условия истинности сложных формул, понятия истинности в модели и $\mathbf{C} \boldsymbol{\Phi}_{\mathbf{O}}^{\mathbf{C}}$-общезначимости стандартные. Доказана адекватность данной семантики сингулярной силлогистике $\mathbf{C} \mathbf{\Phi}_{\mathbf{O}}^{\mathbf{C}}$ :

METATЕОРЕМА 6. Всякая формула доказуема в исчислении $\mathbf{C} \boldsymbol{\Phi}_{\mathbf{O}}^{\mathbf{C}}$, если и только если она является $\mathbf{C} \boldsymbol{\Phi}_{\mathbf{O}}^{\mathbf{C}}$-общезначимой.

Перевод силлогистических формул оккамовского языка осуществляется в язык исчисления предикатов, расширенный за счет введения символа =, причем переводу формул предшествует отображение множества силлогистических терминов в множество предикатов исчисления предикатов с равенством $\left(\mathbf{И П}^{=}\right)$. Эта особенность обусловлена следующим обстоятельством. Принимаемая в фундаментальной силлогистике интерпретация категорических высказываний фиксировалась ранее посредством перевода * на язык логики предикатов. Однако при этом предполагалось, что субъектами и предикатами общих и частных высказываний могут быть только общие термины, которые в логике предикатов выступают в качестве одноместных предикаторов (знаков свойств). В оккамовском силлогистическом языке, как уже говорилось, на местах субъектов и предикатов общих и частных высказываний могут находиться и сингулярные термины. Причем тип их значений в составе множественных высказываний в силлогистике (одноэлементные классы) отличается от типа значений сингулярных терминов в логике предикатов (индивиды). Свойство принадлежности классу, единственным элементом которого является объект v, может быть выражено в ИП $=$ посредством предиката $\ll x=v »$. Очевидно также, что свойство принадлежности классу $S$ предмета х (где $S$ - общий термин) можно выразить посредством предиката «Sx». 
Функция $\theta$, сопоставляющая силлогистическому термину некоторый предикат с одной предметной переменной $x$ в языке ИП=, задается следующим образом:

$$
\theta(v)=\theta(x=v), \quad \theta(S)=S \mathrm{x},
$$

где $v$ - сингулярный, $S$ - общий термин.

Функция * переопределяется так, чтобы она сопоставляла формулам оккамовского силлогистического языка замкнутые формулы языка и выражала принимаемую в фундаментальной силлогистике интерпретацию категорических высказываний $(S$ и $P$ здесь являются произвольными силлогистическими терминами):

$$
\begin{aligned}
& (S \mathbf{a} P)^{*}=\forall x(\theta(S) \supset \theta(P)), \quad(S \mathbf{e} P)^{*}=\forall x(\theta(S) \supset \neg \theta(P)), \\
& (S \mathbf{i} P)^{*}=\exists x(\theta(S) \& \theta(P)), \quad(S \mathbf{o} P)^{*}=\exists x(\theta(S) \& \neg \theta(P)), \\
& (\neg \mathbf{A})^{*}=\neg\left(\mathbf{A}^{*}\right), \quad(\mathbf{A} \nabla \mathbf{B})^{*}=\mathbf{A}^{*} \nabla \mathbf{B}^{*} \text {. }
\end{aligned}
$$

В [2] было доказано, что модифицированный перевод * погружает С $\boldsymbol{\Phi}_{\mathrm{O}}^{\mathrm{C}}$ в ИП $=$ :

МЕТАТЕОРЕМА 7. Всякая формула А доказуема в исчислении $\mathbf{C} \boldsymbol{\Phi}_{\mathbf{O}}^{\mathbf{C}}$, если и только если ее перевод $\mathbf{A}^{*}$ доказуем в ИП $\mathbf{~}^{=}$.

Построим теперь в оккамовском языке систему сингулярной позитивной силлогистики, которая является расширением силлогистики Лукасевича. Назовем эту систему $\mathbf{C 4} \mathbf{O}$. Аксиоматическая формулировка данного исчисления получается за счет добавления к множеству постулатов системы СФ $\mathbf{C}$ новой схемы аксиом:

\section{$\mathbf{A}_{\mathbf{O}}^{\mathbf{C}} 7 . \quad S \mathbf{i} S$.}

Покажем, что система $\mathbf{C 4} \underset{\mathrm{O}}{\mathrm{C}}$ содержит все законы силлогистики Лукасевича:

METATEOPEMA 8. Всякая теорема системъ $\mathbf{C 4}$ доказуема в исчислении $\mathbf{C} 4 \mathbf{O}$.

\section{Доказательство.}

Доказательства в $\mathbf{C 4} \underset{\mathrm{O}}{\mathrm{C}}$ аксиомных схем A1-A6 системы $\mathbf{C 4}$ аналогично их доказательствам в $\mathbf{C} 4_{\mathbf{A}}^{\mathbf{C}}$, представленным в обосновании Метатеоремы 1. Отличие в том, что вместо силлогистической константы ӓ должна стоять $\mathbf{a}$, а все вхождения константы ё должны быть заменены на е. Кроме того, в качестве 
термина $v$, используемого в доказательствах аксиом, выбирается сингулярный термин, который отсутствует в самих этих аксиомах. Остается только доказать в $\mathbf{C} 4_{\mathrm{O}}^{\mathbf{C}}$ аналог используемой в указанных выводах аксиомной схемы $\mathbf{A}_{\mathbf{A}}^{\mathbf{C}} \mathbf{5}(v \ddot{\mathbf{e}} S \equiv \neg v \ddot{\mathbf{a}} S)$ системы $\mathbf{C} 4_{\mathbf{A}}^{\mathbf{C}}-$ формулу $v \mathbf{e} S \equiv \neg v \mathbf{a} S$ :

1. $(v \mathbf{a} S \& v \mathbf{a} v) \supset v \mathbf{i} S \quad \mathbf{A}_{\mathbf{O}}^{\mathbf{C}} 4$

2. $v \mathbf{a} v \quad \mathbf{A}_{\mathbf{O}}^{\mathbf{C}} \mathbf{1}$

3. $v \mathbf{a} S \supset v \mathbf{i} S \quad 1,2 ; \pi \mathbf{B}$

4. $\quad(w \mathbf{a} S \& v \mathbf{a} w) \supset v \mathbf{a} S \quad \mathbf{A}_{\mathbf{O}}^{\mathbf{C}} \mathbf{3}$

5. $w \mathbf{a} v \supset v \mathbf{a} w \quad \mathbf{A}_{\mathbf{O}}^{\mathbf{C}} \mathbf{2}$

6. $\quad(w \mathbf{a} v \& w \mathbf{a} S) \supset v \mathbf{a} S \quad 4,5 ;$ ЛВ

7. $v \mathbf{i} S \supset v \mathbf{a} S \quad 6 ; \mathbf{\Pi 2}$

8. $v \mathbf{e} S \equiv \neg v \mathbf{i} S \quad \mathbf{C A}_{\mathbf{O}}^{\mathbf{C}} \mathbf{5}$

9. $v \mathbf{e} S \equiv \neg v \mathbf{a} S \quad 3,7,8 ;$ ЛВ

Q.E.D.

Имеются веские основания считать именно систему $\mathbf{C 4} \underset{\mathrm{O}}{\mathrm{C}}$ адекватной современной формализацией сингулярного позитивного фрагмента традиционной силлогистики. Действительно, в том варианте силлогистики, который излагается в учебниках по традиционной логике, допускается использование сингулярных терминов на предикатной позиции, а единичные высказывания сводятся к множественным (обычно к общим). Кроме того, с семантической точки зрения, в системе $\mathbf{C} \mathbf{4}_{\mathbf{O}}^{\mathbf{C}}$ принимается, что будет показано ниже, характерная для традиционной версии силлогистики предпосылка о непустоте силлогистических терминов.

Зададим перевод $\Theta^{\prime}$, фиксирующий указанную экзистенциальную предпосылку, формул рассматриваемого силлогистического языка на язык $\mathbf{И П}^{=}$и докажем адекватность данного перевода.

Пусть A - произвольная формула оккамовского варианта языка сингулярной позитивной силлогистики, ${ }^{*}$ - переопределенный только что перевод, погружающий систему СФ $\mathbf{O}_{\text {в }}^{\mathbf{~ И П}}=$. Пусть $S_{1}, \ldots, S_{n}-$ список всех содержащихся в формуле А общих терминов. Если данный список непуст, то

$$
\Theta^{\prime}(\mathbf{A})=\left(\exists x S_{1} x \& \ldots \& \exists x S_{n} x\right) \supset \mathbf{A}^{*} ;
$$

а в случае, когда $\mathbf{A}$ не содержит общих терминов, т.е. силлоги- 
стическими терминами в составе $\mathbf{A}$ являются лишь сингулярные термины, -

$$
\Theta^{\prime}(\mathbf{A})=\mathbf{A}^{*} \text {. }
$$

При переводе в язык ИП $=$ формул языка $\mathbf{C} 4 \mathbf{O}$ снова нет необходимости явно выражать предпосылку о непустоте сингулярных терминов, поскольку формула $\exists x(x=v)$, содержащая информацию о непустоте термина $v$, доказуема в $\mathbf{И П}^{=}$.

В ходе обоснования тезиса о погружаемости $\mathbf{C} 4_{\mathbf{O}}^{\mathbf{C}}$ в ИП ${ }^{=}$посредством $\Theta^{\prime}$, как и в рассмотренном ранее случае первого сингулярного расширения $\mathbf{C 4}$, используем в качестве «промежуточной» фундаментальную силлогистику, на этот раз - систему $\mathbf{C} \Phi_{\mathbf{O}}^{\mathrm{C}}$.

Зададим перевод из $\mathbf{C} 4 \underset{\mathbf{O}}{\mathbf{C}}$ в $\mathbf{C} \boldsymbol{\Phi}_{\mathbf{O}}^{\mathbf{C}}$. Пусть $S_{1}, \ldots, S_{n}-$ список всех общих терминов, содержащихся в произвольной формуле А языка этих силлогистик.

Если данный список непуст, то

$$
\omega^{\prime}(\mathbf{A})=\left(S_{1} \mathbf{i} S_{1} \& \ldots \& S_{n} \mathbf{i} S_{n}\right) \supset \mathbf{A},
$$

а в случае отсутствия универсалий в составе $\mathbf{A}-$

$$
\omega^{\prime}(\mathbf{A})=\mathbf{A}
$$

С использованием сформулированного В.А. Смирновым критерия погружаемости одного исчисления в другое докажем метатеорему:

METATEOPEMA 9. Перевод $\omega^{\prime}$ погружает $\mathbf{C 4} \underset{\mathbf{O}}{\mathbf{C}}$ в $\mathbf{C} \boldsymbol{\Phi}_{\mathbf{O}}^{\mathbf{C}}$.

\section{Доказательство.}

Покажем сначала, что $\omega /$-переводы всех теорем $\mathbf{C 4}$ O доказуемы в $\mathbf{C} \boldsymbol{\Phi}_{\mathbf{O}}^{\mathrm{C}}$.

Рассмотрим доказательство $\mathbf{C}_{1}, \ldots, \mathbf{C}_{k}$ произвольной теоремы системы $\mathbf{C} 4 \mathbf{O}$, и методом возвратной индукции покажем для каждого $\mathbf{C}_{i}$, что $\mathbf{C} \boldsymbol{\Phi}_{\mathbf{O}}^{\mathbf{C}} \vdash \omega^{\prime}\left(\mathbf{C}_{i}\right)$.

Рассмотрим сначала случай, когда $\mathbf{C}_{i}-$ аксиома $\mathbf{C} 4 \mathbf{O}$. Аксиомы $\mathbf{A}_{\mathbf{O}}^{\mathbf{C}} \mathbf{1}$ и $\mathbf{A}_{\mathbf{O}}^{\mathbf{C}} \mathbf{2}$ системы $\mathbf{C} 4 \mathbf{O}_{\mathbf{O}}^{\mathbf{C}}$ не содержат в своем составе общих терминов, их $\omega^{\prime}$-переводы совпадают с ними самими и являются аксиомами $\mathbf{C} \Phi_{\mathbf{O}}^{\mathbf{C}}$. Аксиомы $\mathbf{A}_{\mathbf{O}}^{\mathbf{C}} \mathbf{3}-\mathbf{A}_{\mathbf{O}}^{\mathbf{C}} \mathbf{6}$ системы $\mathbf{C} 4 \mathbf{O}$ являются также аксиомами $\mathbf{C} \boldsymbol{\Phi}_{\mathbf{O}}^{\mathbf{C}}$. Поэтому если они не содержат общих терминов, их $\omega^{\prime}$-переводы доказуемы в $\mathbf{C} \Phi_{\mathbf{O}}^{\mathbf{C}}$. Если же эти аксиомы содержат в своем составе общие термины, их $\omega^{\prime}$-переводы 
выводятся в последней системе из соответствующих аксиом с использованием частного случая закона утверждения консеквента $-\mathbf{C}_{i} \supset\left(\left(S_{1} \mathbf{i} S_{1} \& \ldots \& S_{n} \mathbf{i} S_{n}\right) \supset \mathbf{C}_{i}\right) \cdot \omega^{\prime}$-перевод аксиомы $\mathbf{A}_{\mathbf{O}}^{\mathbf{C}} \mathbf{7}$ $S \mathbf{i} S$, если $S$ - общий термин, является законом логики высказываний $S \mathbf{i} S \supset S \mathbf{i} S$. Если же $S-$ сингулярный термин, например $v$, то $\omega^{\prime}$-перевод данной аксиомы есть формула $v \mathbf{i} v$, которая доказуема в системе $\mathbf{C} \boldsymbol{\Phi}_{\mathbf{O}}^{\mathbf{C}}$ :
1. $v \mathbf{a} v$
$\mathbf{A}_{\mathrm{O}}^{\mathrm{C}} \mathbf{1}$
2. $\quad(v \mathbf{a} v \& v \mathbf{a} v) \supset v \mathbf{i} v$
$\mathbf{A}_{\mathrm{O}^{4}}^{\mathrm{C}}$
3. $v \mathbf{i} v$
1,$2 ;$ ЛВ

Пусть $\mathbf{C}_{i}$ в составе доказательства $\mathbf{C}_{1}, \ldots, \mathbf{C}_{k}$ в системе $\mathbf{C} \mathbf{4}_{\mathbf{O}}^{\mathbf{C}}$ получена по правилу modus ponens из предыдущих формул этой последовательности $\mathbf{C}_{j} \supset \mathbf{C}_{i}$ и $\mathbf{C}_{j}$. По индуктивному допущению, $\mathbf{C} \boldsymbol{\Phi}_{\mathbf{O}}^{\mathbf{C}} \vdash \omega \prime\left(\mathbf{C}_{j} \supset \mathbf{C}_{i}\right)$ и $\mathbf{C} \boldsymbol{\Phi}_{\mathbf{O}}^{\mathbf{C}} \vdash \omega^{\prime}\left(\mathbf{C}_{j}\right)$. Рассмотрим четыре случая в зависимости от того, содержатся ли в составе формул $\mathbf{C}_{j}$ и $\mathbf{C}_{i}$ общие термины.

1. Ни $\mathbf{C}_{j}$, ни $\mathbf{C}_{i}$ не содержат общих терминов. Тогда $\omega^{\prime}\left(\mathbf{C}_{j} \supset\right.$ $\left.\mathbf{C}_{i}\right)=\mathbf{C}_{j} \supset \mathbf{C}_{i}, \omega^{\prime}\left(\mathbf{C}_{j}\right)=\mathbf{C}_{j}$. Формула $\omega^{\prime}\left(\mathbf{C}_{i}\right)$, совпадающая с $\mathbf{C}_{i}$, может быть получена в системе $\mathbf{C} \boldsymbol{\Phi}_{\mathbf{O}}^{\mathbf{C}}$ по modus ponens.

2. $\mathbf{C}_{j}$ не содержит, а $\mathbf{C}_{i}$ содержит общие термины (например, $\left.S_{1}, \ldots, S_{n}\right)$. В этом случае $\omega^{\prime}\left(\mathbf{C}_{j} \supset \mathbf{C}_{i}\right)=\left(S_{1} \mathbf{i} S_{1} \& \ldots \&\right.$ $\left.S_{n} \mathbf{i} S_{n}\right) \supset\left(\mathbf{C}_{j} \supset \mathbf{C}_{i}\right)$, а $\left(\mathbf{C}_{j}\right)=\mathbf{C}_{j}$. Формула $\left(\mathbf{C}_{i}\right)$, совпадающая с $\left(S_{1} \mathbf{i} S_{1} \& \ldots \& S_{n} \mathbf{i} S_{n}\right) \supset \mathbf{C}_{i}$, выводится в $\mathbf{C} \Phi_{\mathbf{O}}^{\mathbf{C}}$ из этих двух теорем с использованием закона коммутации и правила modus ponens.

3. $\mathbf{C}_{i}$ не содержит, а $\mathbf{C}_{j}$ содержит универсалии $\left(S_{1}, \ldots, S_{n}\right)$. Тогда $\omega^{\prime}\left(\mathbf{C}_{j} \supset \mathbf{C}_{i}\right)=\left(S_{1} \mathbf{i} S_{1} \& \ldots \& S_{n} \mathbf{i} S_{n}\right) \supset\left(\mathbf{C}_{j} \supset \mathbf{C}_{i}\right)$, а $\omega^{\prime}\left(\mathbf{C}_{j}\right)=\left(S_{1} \mathbf{i} S_{1} \& \ldots \& S_{n} \mathbf{i} S_{n}\right) \supset \mathbf{C}_{j}$. С использованием закона самодистрибутивности импликации из этих двух формул выводим в $\mathbf{C} \boldsymbol{\Phi}_{\mathbf{O}}^{\mathbf{C}}$ формулу $\left(S_{1} \mathbf{i} S_{1} \& \ldots \& S_{n} \mathbf{i} S_{n}\right) \supset \mathbf{C}_{i}$. Рассуждением от противного покажем, что и формула $\mathbf{C}_{i}$ в этом случае будет теоремой $\mathbf{C} \boldsymbol{\Phi}_{\mathbf{O}}^{\mathbf{C}}$. Допустим, что это не так. Тогда в силу Метатеоремы $7 \mathbf{C}_{i}$ не является $\mathbf{C} \boldsymbol{\Phi}_{\mathbf{O}^{-}}^{\mathbf{C}}$ общезначимой, т.е. найдется модель $\langle\mathbf{D}, \varphi\rangle$, в которой 
$\mathbf{C}_{i}$ ложна. Пусть функция означивания $\varphi^{\prime}$ всем силлогистическим терминам, кроме общих терминов $S_{1}, \ldots, S_{n}$, сопоставляет те же значения, что и $\varphi$, а $\varphi^{\prime}\left(S_{1}\right)=\cdots=$ $\varphi^{\prime}\left(S_{n}\right)=\mathbf{D}$. В силу непустоты множества $\mathbf{D}$ каждая из формул $S_{1} \mathbf{i} S_{1}, \ldots, S_{n} \mathbf{i} S_{n}$, а значит и их конъюнкция, истинны в модели $<\mathbf{D}, \varphi^{\prime}>$. Поскольку $\mathbf{C}_{i}$ не содержит общих терминов $S_{1}, \ldots, S_{n}$, она будет иметь в $<\mathbf{D}, \varphi^{\prime}>$ то же значение, что и в $\langle\mathbf{D}, \varphi\rangle$, а именно значение «ложь». Следовательно, формула $\left(S_{1} \mathbf{i} S_{1} \& \ldots \& S_{n} \mathbf{i} S_{n}\right) \supset \mathbf{C}_{i}$ ложна в $\left\langle\mathbf{D}, \varphi^{\prime}\right\rangle$. Тогда она не является $\mathbf{C} \boldsymbol{\Phi}_{\mathbf{O}}^{\mathbf{C}}$-общезначимой, и по Метатеореме 7, не доказуема в исчислении $\mathbf{C} \boldsymbol{\Phi}_{\mathbf{O}}^{\mathbf{C}}$. Последнее утверждение вступает в противоречие с ранее полученным. Таким образом, допущение о недоказуемости формулы $\mathbf{C}_{i}$ в $\mathbf{C} \boldsymbol{\Phi}_{\mathbf{O}}^{\mathbf{C}}$ опровергнуто, а в данном случае $\mathbf{C}_{i}$ совпадает с $\omega^{\prime}\left(\mathbf{C}_{i}\right)$. Поэтому $\mathbf{C} \boldsymbol{\Phi}_{\mathbf{O}}^{\mathbf{C}} \vdash \omega^{\prime}\left(\mathbf{C}_{i}\right)$.

4. Обе формулы $-\mathbf{C}_{j}$ и $\mathbf{C}_{i}-$ содержат в своем составе общие термины. Обосновываем доказуемость $\omega^{\prime}\left(\mathbf{C}_{i}\right)$, рассуждая аналогично тому, как при доказательстве Метатеоремы 4.

Пусть $\mathbf{C}_{i}$ получено из $\mathbf{C}_{j}$ по правилу П2. Тогда $\mathbf{C}_{j}=(v \mathbf{a} S \&$ $v \mathbf{a} P) \supset \mathbf{A}$, а $\mathbf{C}_{i} \mp S \mathbf{i} P \supset \mathbf{A}$. Очевидно, что множества универсалий в $\mathbf{C}_{j}$ и $\mathbf{C}_{i}$ совпадают. Если эти множества пусты, то $\omega_{1}\left(\mathbf{C}_{i}\right)$, совпадающая с $\mathbf{C}_{i}$, выводится в системе $\mathbf{C} \boldsymbol{\Phi}_{\mathbf{O}}^{\mathbf{C}}$ из $\omega_{1}\left(\mathbf{C}_{j}\right)$, совпадающей с $\mathbf{C}_{j}$, по правилу П2. Если же $\mathbf{C}_{j}$ содержит в своем составе универсалии $S_{1}, \ldots, S_{n}$, рассуждаем как при рассмотрении случая $\mathbf{R 2}$ Метатеоремы 4 (меняя в самом рассуждении константу ӓ на $\mathbf{a}$, а в его анализе ссылку на правило $\mathbf{R} \mathbf{2}$ ссылкой на применение П2).

Аналогичным образом рассматривается случай, когда $\mathbf{C}_{i}$ получено из $\mathbf{C}_{j}$ по правилу ПЗ

Индуктивное рассуждение завершено. Показано, что $\omega^{\prime}$-перевод любой теоремы $\mathbf{C} \mathbf{4}_{\mathbf{O}}^{\mathbf{C}}$ доказуем в системе $\mathbf{C} \boldsymbol{\Phi}_{\mathbf{O}}^{\mathbf{C}}$.

Задаем обратный перевод $\omega^{\prime \prime}$ из $\mathbf{C} \Phi_{\mathbf{O}}^{\mathrm{C}}$ в $\mathbf{C} 4_{\mathbf{O}}^{\mathrm{C}}$ :

$$
\omega^{\prime \prime}(\mathbf{A})=\mathbf{A} .
$$

Поскольку исчисление $\mathbf{C} \boldsymbol{\Phi}_{\mathrm{O}}^{\mathrm{C}}$ является подсистемой $\mathbf{C} 4 \mathbf{\mathrm { O }}, \omega^{\prime \prime}$ перевод любой теоремы $\mathbf{C} \boldsymbol{\Phi}_{\mathbf{O}}^{\mathbf{C}}$ доказуем в $\mathbf{C} 4_{\mathbf{O}}^{\mathbf{C}}$. 
Остается удостовериться в выполнении третьей части критерия погружаемости В.А. Смирнова - в том, что $\mathbf{C} 4 \mathbf{O}_{\vdash}^{\mathbf{C}} \vdash \mathbf{A} \equiv$ $\omega^{\prime \prime}\left(\omega^{\prime}(\mathbf{A})\right)$ для любой формулы $\mathbf{A}$. Если $\mathbf{A}$ не содержит общих терминов, то $\omega^{\prime \prime}\left(\omega^{\prime}(\mathbf{A})\right)=\mathbf{A}$, а $\mathbf{A} \equiv \mathbf{A}-$ теорема $\mathbf{C} 4 \mathbf{O}$. Если же $\mathbf{A}$ содержит общие термины, то формула $\mathbf{A} \equiv \omega^{\prime \prime}\left(\omega^{\prime}(\mathbf{A})\right)$ доказывается в $\mathbf{C} 4 \mathbf{O}$ так же, как и в Метатеореме 4.

Q.E.D.

METATEOPEMA 10. Перевод $\Theta^{\prime}$ погружает $\mathbf{C 4} \mathbf{O}_{\text {в }}^{\mathbf{C}} \mathbf{\Pi}^{=}$.

Доказательство. Из утверждения о погружаемости СФ $\boldsymbol{\Phi}_{\mathrm{O}}^{\mathrm{C}}$ ИП $=$ посредством модифицированной функции * (Метатеорема 7) и только что доказанной погружаемости $\mathbf{C} 4 \underset{\mathrm{O}}{\mathrm{C}}$ в $\mathbf{C} \boldsymbol{\Phi}_{\mathrm{O}}^{\mathrm{C}}$ посредством $\omega^{\prime}$ (Метатеорема 9) следует, что система $\mathbf{C} 4 \underset{\mathbf{O}}{\mathbf{C}}$ погружается в ИП= посредством композиции $\omega^{\prime}$ и $^{*}$. Данная композиция равносильна в ИП $=$ переводу $\Theta^{\prime}$. Если силлогистическая формула $\mathbf{A}$ не содержит общих терминов, то $\left[\omega^{\prime}(\mathbf{A})\right]^{*}=\mathbf{A}^{*}=\Theta^{\prime}(\mathbf{A})$. B противном случае осуществляем рассуждение, аналогичное содержащемуся в доказательстве Метатеоремы 5.

Q.E.D.

\section{Литература}

[1] Лукасевич Я. Аристотелевская силлогистика с точки зрения современной формальной логики. М.: Издательство иностранной литературы, 1959.

[2] Маркин В.И. Силлогистические теории в современной логике. М.: Издательство МГУ, 1991.

[3] Смирнов B.A. Адекватный перевод утверждений силлогистики в исчисление предикатов // Актуальные проблемы логики и методологии науки. Киев: Наукова думка, 1980.

[4] Смирнов B.A. Логические методы анализа научного знания. М.: Наука, 1987. 